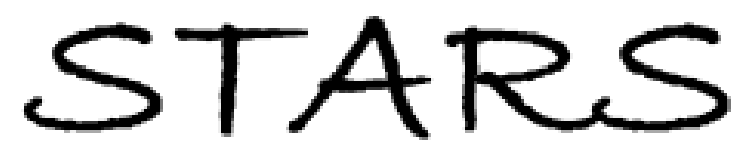

University of Central Florida

STARS

$1-1-2014$

\title{
Simultaneous low noise radio frequency tone and narrow linewidth optical comb generation from a regeneratively mode- locked laser
}

Ibrahim Ozdur

Sarper Ozharar

Peter J. Delfyett

University of Central Florida

Find similar works at: https://stars.library.ucf.edu/facultybib2010

University of Central Florida Libraries http://library.ucf.edu

This Article is brought to you for free and open access by the Faculty Bibliography at STARS. It has been accepted for inclusion in Faculty Bibliography 2010s by an authorized administrator of STARS. For more information, please contact STARS@ucf.edu.

\section{Recommended Citation}

Ozdur, Ibrahim; Ozharar, Sarper; and Delfyett, Peter J., "Simultaneous low noise radio frequency tone and narrow linewidth optical comb generation from a regeneratively mode-locked laser" (2014). Faculty Bibliography 2010s. 5922.

https://stars.library.ucf.edu/facultybib2010/5922

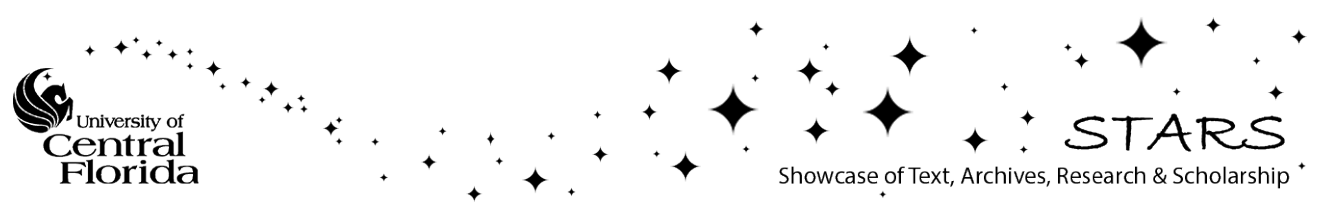




\section{Optical Engineering}

\section{Simultaneous low noise radio frequency tone and narrow linewidth optical comb generation from a regeneratively mode-locked laser}

Ibrahim Ozdur Sarper Ozharar Peter J. Delfyett 


\section{Simultaneous low noise radio frequency tone and narrow linewidth optical comb generation from a regeneratively mode-locked laser}

\author{
Ibrahim Ozdur, ${ }^{\text {a }}$ ( Sarper Ozharar, ${ }^{b}$ and Peter J. Delfyett ${ }^{c}$ \\ aAbdullah Gul University, Faculty of Engineering and \\ Natural Sciences, Melikgazi, Kayseri 38080, Turkey \\ ${ }^{\mathrm{b}}$ Bahçeşehir University, Faculty of Arts and Sciences, \\ Besiktas, Istanbul 34353, Turkey \\ 'University of Central Florida, CREOL and FPCE: The \\ College of Optics and Photonics, Orlando, Florida 32816, \\ United States
}

\begin{abstract}
A regeneratively mode-locked laser with simultaneous low noise radio frequency (RF) tone and optical comb generation is presented. The laser does not need any external RF signal and emits a pulse train at $\sim 10 \mathrm{GHz}$ repetition rate with a 1.5-ps optical pulse width after compression. The generated RF tone has a signal-to-noise ratio of $121 \mathrm{~dB} / \mathrm{Hz}$ and an $\mathrm{RF}$ fluctuation of $10^{-9}$ over $0.1 \mathrm{~s}$. The optical frequency comb spacing is also at $\sim 10 \mathrm{GHz}$ and the optical comb tooth has a linewidth of $<1 \mathrm{kHz}$. () 2014 Society of Photo-Optical Instrumentation Engineers (SPIE) [DOI: 10.1117/1.OE.53.10.100501]
\end{abstract}

Keywords: fiber lasers; Fabry-Perot; mode locking; laser linewidth. Paper 141150L received Jul. 18, 2014; revised manuscript received Sep. 15, 2014; accepted for publication Oct. 7, 2014; published online Oct. 29, 2014.

\section{Introduction}

Optical frequency stabilized mode-locked lasers at high repetition rates have a wide variety of applications such as in radar systems, coherent communication, signal processing including optical code division multiple access, dense wavelength-division-multiplexing, and optical arbitrary waveform generation. $\mathbb{Q}^{\mathrm{T}}$ The performance of such systems is generally limited by the timing jitter and optical wavelength/frequency stability of the source. Actively mode locked lasers driven by ultralow noise radio frequency (RF) oscillators have excellent RF and optical noise performances. However, such ultralow noise RF oscillators are expensive, power inefficient, and heavy, limiting their usage in weight-critical systems such as satellites. The ultralow noise RF oscillator can be eliminated via the regeneratively mode-locked laser configuration. A semiconductor-based $10 \mathrm{GHz}$ repetition rate regeneratively mode-locked laser with $121 \mathrm{dBc} / \mathrm{Hz}$ signalto-noise ratio at $\mathrm{MHz}$ offset frequencies and less than $1 \mathrm{kHz}$ optical linewidth is reported in this work.

*Address all correspondence to: Ibrahim Ozdur, E-mail: ibrahim.ozdur@agu .edu.tr
High quality (Q)-factor oscillators have lower phase noise and timing jitter. Mode-locked lasers with high-Q factors can be obtained by inserting optical delay lines in the laser cavity ${ }^{\mathrm{E}}$ However, long delay lines reduce the cavity fundamental frequency. If a high repetition rate mode-locked laser is needed, the repetition rate of the long cavity mode-locked laser can be set to a multiple integer of the cavity fundamental frequency, which is called harmonic mode locking. One drawback of harmonic mode locking is the generated supermode noise, originating from the competition among multiple supermode families. One way to suppress the supermode noise is to use an intracavity Fabry-Perot etalon. 0 The intracavity etalon eliminates all but one optical supermode family by periodic filtering. In practice, the maximum cavity length is limited by the transmission window widths of the etalon.

The optical comb lines and etalon transmission peaks can drift over time due to environmental effects which would destroy the stability of mode locking. In order to minimize the environmental effects, the optical frequency needs to be locked to the etalon transmission window peaks. The best method to lock the optical frequency to an etalon transmission window is the Pound-Drever-Hall $(\mathrm{PDH})$ technique. $\mathrm{A}$ 1000 finesse etalon is used in this work which is a factor 10 increase over the previous one. This allowed us to have a 10 times narrower etalon transmission window and hence a 10 times sharper PDH error signal and tighter optical frequency lock.

\section{Experiment}

The regeneratively mode-locked laser (Fig. (1) has three main loops labeled as the laser cavity, RF feedback, and PDH feedback loops. The laser cavity consists of a semiconductor optical amplifier (SOA), a free space etalon with $10.287 \mathrm{GHz}$ free spectral range (FSR), a variable optical delay to tune the cavity length, and an intensity modulator. Two isolators, before and after the SOA, maintain the unidirectional operation of the laser. The RF feedback loop has a photodetector, an RF phase shifter, and an RF amplifier. A portion of the laser output pulse train is photodetected, amplified, and then sent to the intensity modulator as the driving signal. The laser cavity and the RF feedback loop lengths are tuned so that both match the etalon FSR. The last part in the regeneratively mode-locked laser schematic is the PDH loop, which is necessary to lock the optical frequency to the resonance peaks of the etalon. Part of the output optical signal is phase modulated at $640 \mathrm{MHz}$ and then sent back to the intracavity etalon perpendicular to the polarization state of the optical signal. The reflected beam from the etalon is separated from the optical signal by a polarization beam splitter (PBS). This reflection signal is mixed with the local oscillator after photodetection. The obtained PDH error signal is sent to a proportional-integral-differential (PID) controller, which readjusts the optical delay length via a piezoelectric optical phase shifter to minimize the error signal and stabilizes the optical frequency.

\section{Results}

The generated RF tone of the regeneratively mode-locked laser, shown in Fig. 2, is centered at $10.287 \mathrm{GHz}$ which is defined by the FSR of the intracavity etalon. The laser

0091-3286/2014/\$25.00 (C) 2014 SPIE 


\section{OE Letters}

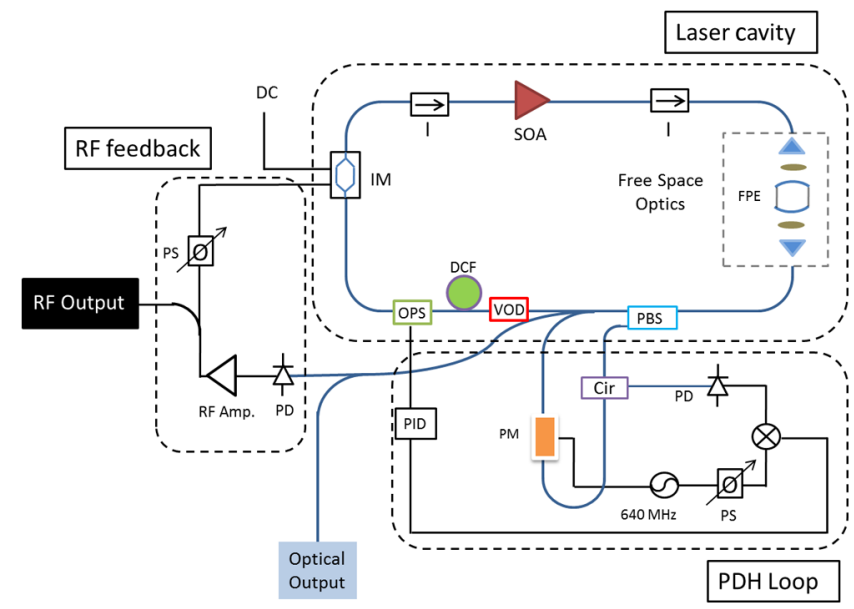

Fig. 1 A complete setup of the regeneratively mode-locked laser. The laser cavity, PDH loop, and RF feedback loop can be seen in dashed rectangles. Some parts in the figure are: I: Optical isolator, PS: RF phase shifter, IM: intensity modulator, PBS: polarization beam splitter, DCF: dispersion-compensating fiber, FPE: Fabry-Perot etalon, VOD variable optical delay, PID: proportional-integral-differential controller, PM: phase modulator, Cir: optical circulator, and OPS: optical phase shifter.

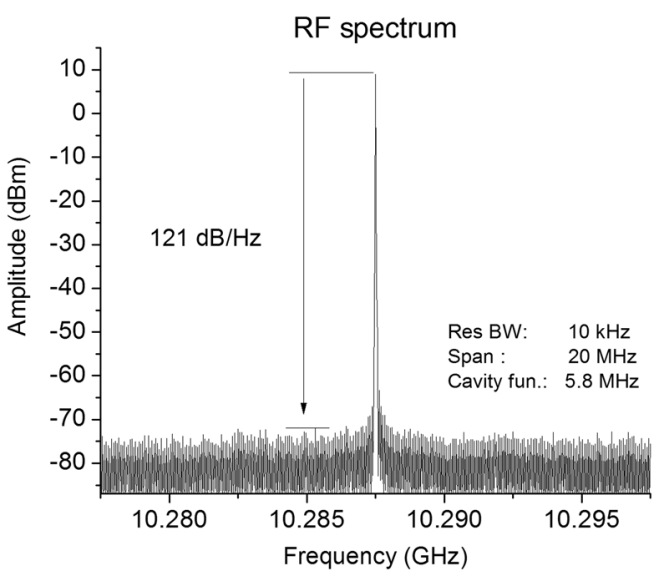

Fig. 2 RF spectrum trace of the RF output.

cavity fundamental frequency is $5.8 \mathrm{MHz}$. Since a phase noise measurement was not available at the time, we analyze the RF spectrum to deduce certain aspects of the noise characteristics of the system. Because no supermode spurs are observed in Fig. \& we can conclude that the supermode

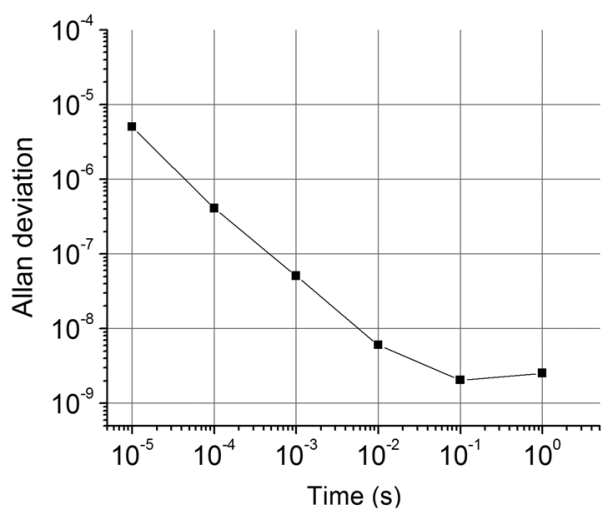

Fig. 3 Allan deviation measurement of the RF tone.

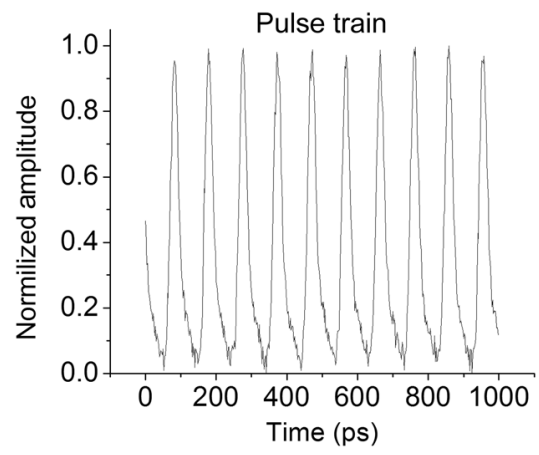

Fig. 4 Optical pulse train of the mode-locked laser-no averaging.

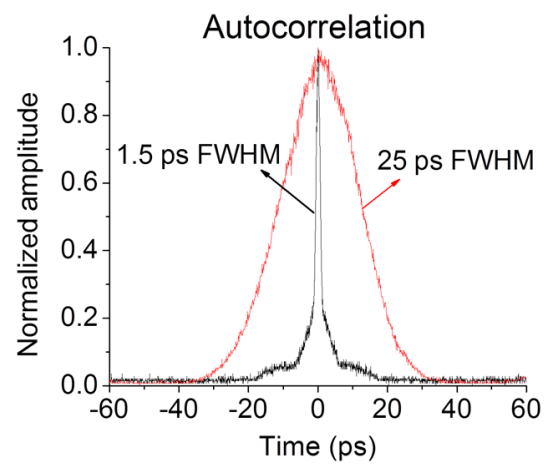

Fig. 5 Optical pulse intensity autocorrelation, directly from the laser (red) and after compression (black).

spurs of the harmonically mode-locked laser are suppressed below $121 \mathrm{~dB} / \mathrm{Hz}$, which is limited by the noise level of the $\mathrm{RF}$ spectrometer. In order to further analyze the RF tone, the Allan deviation is also measured and an RF frequency stability of nearly $10^{-9}$ over $0.1 \mathrm{~s}$ is obtained (Fig. B).

The sampling scope trace of the pulse train without averaging is shown in Fig. 田.

The pulses produced by the mode-locked laser are chirped and have an intensity autocorrelation full-width at half maximum of 25 ps (Fig. 5). The chirped pulses are compressed using a dual grating compressor. The intensity autocorrelation width of the optical pulses is decreased to 1.5 from $25 \mathrm{ps}$ after compression.

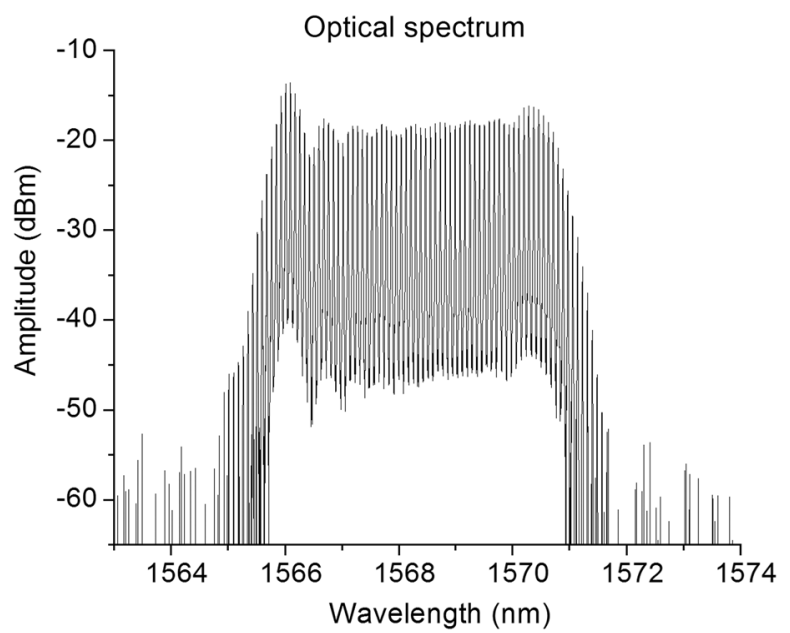

Fig. 6 The optical spectrum of the regeneratively mode-locked laser. 


\section{OE Letters}

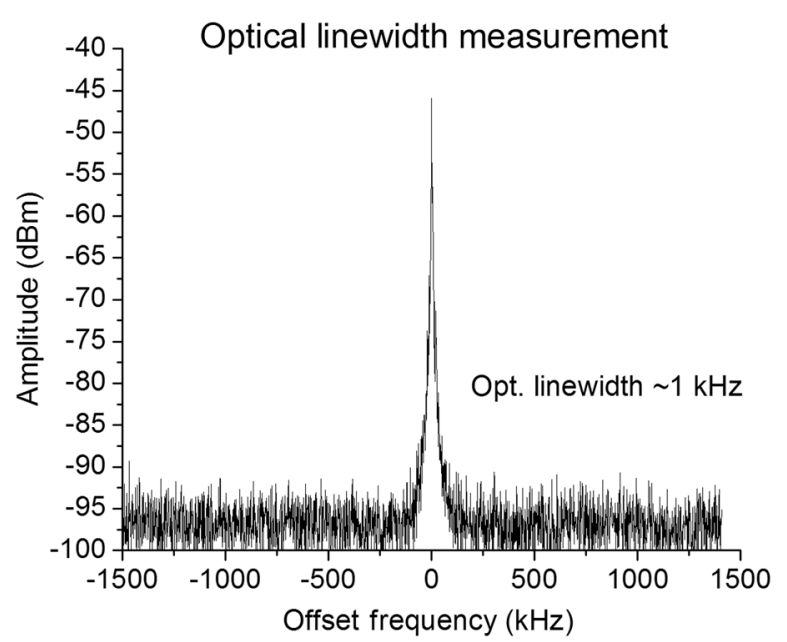

Fig. 7 Optical linewidth measurement of a single optical comb tooth.

The optical spectrum of the regeneratively mode-locked laser is shown in Fig. 6. The optical spectrum's $-10 \mathrm{~dB}$ bandwidth is larger than $5 \mathrm{~nm}$. The visibility of the optical comb lines is nearly $30 \mathrm{~dB}$ and is limited by the resolution of the optical spectrum analyzer. In order to obtain the optical signal-to-noise ratio and the optical linewidth, a heterodyne mixing technique is used. The output of the mode-locked laser is mixed with a commercially available ultranarrow linewidth $(\sim 1 \mathrm{kHz}) \mathrm{CW}$ laser and the width of the resulting beat tone is measured. The optical signal-to-noise ratio is measured to be better than $50 \mathrm{~dB}$ and the linewidth of the optical comb line is $<1 \mathrm{kHz}$ (Fig. \). The narrow optical linewidth is obtained via a tight PDH lock.

\section{Conclusion}

In this work, we have demonstrated a $10.287 \mathrm{GHz}$, semiconductor-based regeneratively mode-locked laser with a 1000 finesse intracavity etalon. The laser generates simultaneous low noise RF tone and an optical frequency comb referenced to the intracavity etalon. The generated RF tone has a supermode noise suppression of $>121 \mathrm{~dB} / \mathrm{Hz}$ and the optical frequency comb has an optical linewidth of less than $1 \mathrm{kHz}$.

\section{References}

1. P. Ghelfi et al., "A fully photonics-based radar system," Nature 507, 341-345 (2014).

2. F. M. Soares et al., "Monolithic InP 100-channel \times 10-GHz device for optical arbitrary waveform generation," IEEE Photonics J. 3(6), 975-985 (2011).

3. P. J. Delfyett et al., "Advanced ultrafast technologies based on optical frequency combs," IEEE J. Sel. Top. Quantum Electron. 18(1), 258-274 (2012).

4. J. Chou, Y. Han, and B. Jalali, "Adaptive RF-photonic arbitrary waveform generator," IEEE Photonics Technol. Lett. 15(4), 581-583 (2003).

5. I. Ozdur et al., "A semiconductor-based $10-\mathrm{GHz}$ optical comb source with sub 3-fs shot-noise-limited timing jitter and $\backslash$ sim $500-\mathrm{Hz}$ comb linewidth," IEEE Photonics Technol. Lett. 22(6), 431-433 (2010).

6. T. Yilmaz, C. M. DePriest, and P. J. Delfyett, "Complete noise characterisation of external cavity semiconductor laser hybridly modelocked at $10 \mathrm{GHz}, "$ Electron. Lett. 37(22), 1338-1339 (2001).

7. F. Quinlan et al., "Self-stabilization of the optical frequencies and the pulse repetition rate in a coupled optoelectronic oscillator," J. Lightwave Technol. 26(15), 2571-2577 (2008).

8. R. W. P. Drever et al., "Laser phase and frequency stabilization using an optical resonator," Appl. Phys. B 31(2), 97-105 (1983). 